\title{
Répartition des foraminifères benthiques dans la zone sud-ouest du système estuarien-lagunaire d'Iguape-Cananéia (Brésil)
}

\author{
Beatriz Beck EICHLER ${ }^{1}$, Jean-Pierre DEBENAY ${ }^{2}$; Carla BONETTI $^{1}$, Wania DULEBA ${ }^{1}$ \\ ${ }^{1}$ Instituto Oceanográfico da Universidade São Paulo \\ (Caixa Postal 66149, 05389-970, São Paulo, SP, Brasil) \\ ${ }^{2}$ Laboratoire de Géologie, Université d'Angers \\ (2 Bd Lavoisier, 49045 Angers cedex, France)
}

- Abstract: One hundred species of benthic foraminifera were found in the study area $\left(25^{\circ} \mathrm{S}-48^{\circ} \mathrm{W}\right)$. The surface sediment of this lagoon contains from 0 to 3,000 tests per $50 \mathrm{~cm}^{3}$ and up to 32 species. The foraminifera fauna of the lagoonal system of Iguape-Cananéia is the richest among those described in other paralic environments of Brazil. The richness of foraminifera fauna can result from the strong marine influence in the Baía de Trapandé. The foraminifera distribution in the study area is characterized by the occurrence of marine assemblages nearby the mouth, with a gradual increase in agglutinating species, such as, Ammotium salsum and Gaudryina exilis. In the confined areas, with low salinity, the assemblages are oligospecific and Milammina earlandi is dominant. The abundance of Pararotalia in the Mar de Cananćia shows that this environment is less restricted than Mar de Cubatão, where the genus is absent. The distribution of foraminifera species shown to be also influenced by urbain sewages.

- Resumo: Cem espécies de foraminíferos bentônicos foram determinadas na area de estudo $\left(25^{\circ} \mathrm{S}-48^{\circ} \mathrm{W}\right)$. $\mathrm{Em} 50 \mathrm{~cm}^{3}$ de sedimento de superfície foram encontradas 0 a 3.000 testas e até 32 espécies. A microfauna do complexo lagunar-estuarino de Iguape-Cananéia é a mais rica dentre aquelas descritas em outros ambientes paralicos do Brasil. Esta riqueza microfaunística seria resultante da grande influência marinha na Baía de Trapandé. A distribuição dos foraminíferos na área estudada é caracterizada pela ocorrência, nas proximidades da desembocadura, de faunas com afinidades marinhas. dominadas por espécies calcarias, que passam a faunas onde as espécies aglutinantes são cada vez mais importantes, como por exemplo, Ammotium salsum e Gaudryina exilis. Finalmente, nas zonas confinadas, com salinidade baixa, as associaçōes sāo oligoespecíficas, e há o predomínio de Milammina earlandi. A abundância do gênero Pararotalia no Mar de Cananéia revela que este é menos confinado que o Mar de Cubatão, onde este gênero é ausente. A distribuição das espécies de foraminiferos é também influenciada pelos efluentes urbanos.

- Descriptors: Foraminifers, Atlantic ocean, Lagoon, Mangrove, Paralic environment, Confinement, Cananćia, São Paulo, Brazil.

- Descritores: Foraminiferos, Oceano Atlântico, Laguna, Mangue, Meios paralicos, Confinamento, Cananéia, SP, Brasil.

\section{Introduction}

Au cours des années 1960, une séric d'ćtudes sur les microfaunes de foraminifères des environnements paraliques de la côte sud du Brésil a été publiée par

Contr. $n^{2} 773$ do Inst. oceanogr. da Usp. l'équipe du Departamento de Paleontologia e Estratigrafia do Instituto de Geociências da Universidade do Rio Grande do Sul (Closs, 1963; 1964; Closs \& Madcira, 1962; 1966; 1967; 1968; Closs \& Medeiros, 1965; 1967; Forti \& Roettger, 1967; Madeira, 1969). Madeira Falceta (1974) a publié une synthèse de ces travaux. Plusieurs études ont également été réalisées dans l'état de Rio de Janeiro 
(Tinoco, 1958; 1966; Zaninetti et al., 1977; 1979; Brönnimann, 1978; 1979; 1980; Brönnimann \& Beurlen, 1977a; 1977b; Brönnimann et al., 1981; Brönnimann \& Dias-Brito, 1982; Beurlen \& Hilterman, 1983). Scott et al. (1990), enfin, ont décrit une association de foraminiferes de la baia de Guaratuba (état de Paraná). Cependant, aucune étude spécifique n'a été consacrée aux foraminiferes actuels des milieux paraliques de l'état de São Paulo. Toutefois, les environnements paraliques de cette région présentent un intérêt tout particulier car:

1) ils se situent à la limite sud de l'extension de la mangrove;

2) ils montrent tous les stades de la dégradation sous l'effet des activétés humaines, depuis des milieux quasiment intacts, jusqu' à des zones totalement dévastées comme c'est le cas dans la mangrove de Santos.

Cette étude est la première d'une sćrie dont l'objectif est de définir, à partir des peuplements de foraminifères, des biomarqueurs d'environnement, permettant :

1) de suivre l'évolution historique des milieux paraliques depuis leur remplissage holocène;

2) de définir un bioindicateur permettant une surveillance continue et à faible coût, de l'impact de l'activété humaine sur ces environnements.

Dans un premier temps, il est nécessaire d'établir un inventaire des peuplements de foraminiferes dans un environnement peu perturbé, de définir leur répartition et de situer ces résultats par rapport à ceux qui ont déjà été obtenus dans les régions voisines du Brésil.

Le complexe laguno-estuarien d'Iguape-Cananéia (Fig. 1), situé au Sud de l'état de São Paulo a été l'objet de nombreuses études géologiques, hydrologiques et biologiques. Des données abondantes sont maintenant disponibles sur son évolution géologique (Petri \& Suguio, 1973 ; Suguio et al., 1976; Martin et al., 1984; 1987), son hydrologie et son hydrodynamique (Suguio \& Tessler, 1983; Por et al., 1984; Mishima et al., 1985 ; SchaefferNovelli et al., 1990; Tessler \& Mahiques, 1993), ses caractéristiques physico-chimiques (Miyao et al., 1986) et ses caractéristiques biologiques (Tundisi, 1981; Carmouze et al., 1986; Kutner \& Aidar-Aragāo, 1986). Cependant, les Foraminifères, micro-organismes très sensibles aux caractéristiques de l'environnement et excellents bioindicateurs, n'ont été que très peu étudiés. Scule, une publication de Petri \& Suguio (1973) décrit les associations observées dans deux carottages et indique succinctement la répartition de quelques genres dans 5 ou 6 échantillons. Petri (1971) décrit quelques spécimens d'Ammonia récoltées à Cananéia.

Le but de ce travail est d'ćtablir un premier inventaire des foraminifères benthiques peuplant la lagune et de décrire la répartition de ces micro-organismes dans la Baía de Trapandé et dans le Sud du Mar de Cubatāo et de Mar de Cananéia. Ces résultats seront ensuite discutés en fonction des données déjà publiées sur les environnements paraliques des côtes du Brésil, au Sud de Rio de Janeiro.

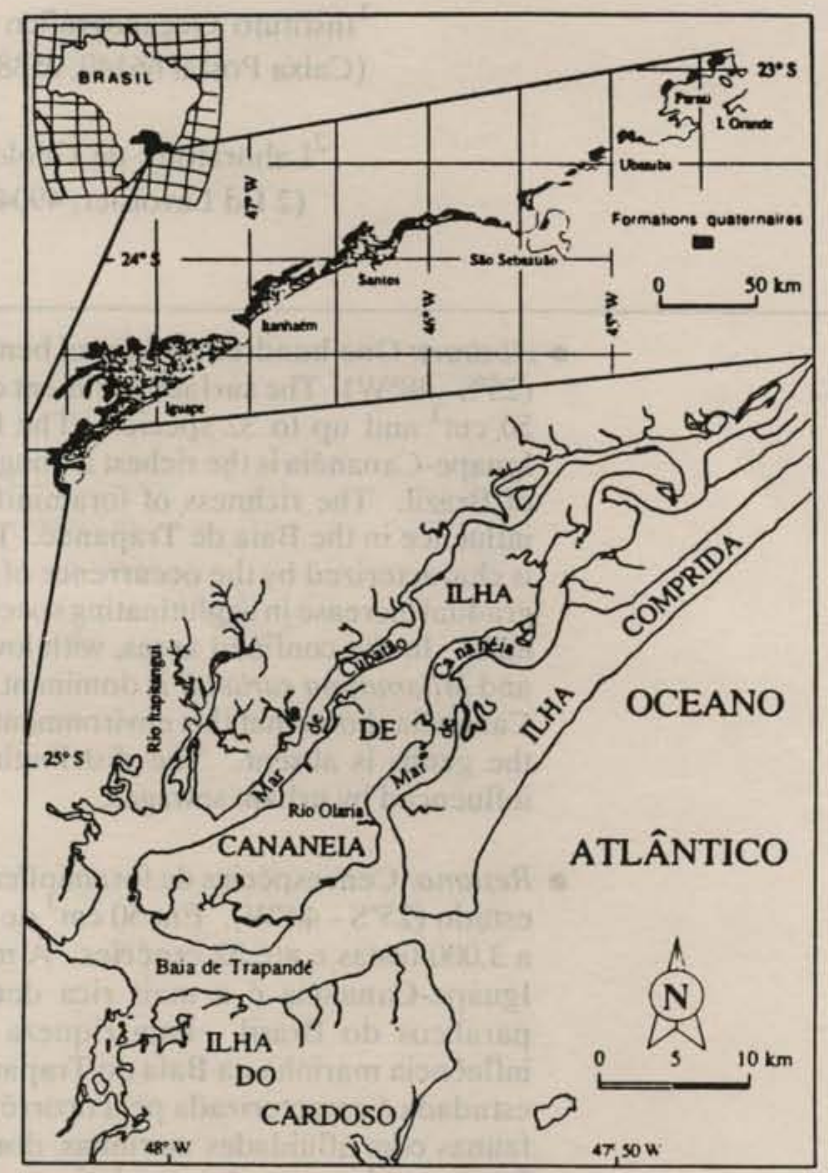

Fig. 1. Localisation géographique de la zone

\section{Matériels et méthodes}

La campagne de prélèvements a eu lieu en octobre 1993. Trente stations ont été retenues, dont une $\left(15^{\prime}\right)$ correspondant à un prélèvement sur des racines de palétuvier (Fig. 2). A chaque station ont été mesurés, la température, la salinćté et le $\mathrm{pH}$ des eaux de fond et de surface. La transparence de l'eau a été évaluéc à l'aide du disque de Secchi.

Le sédiment destiné à l'étude des foraminiferes a été prélevé à la benne. Seuls, les $5 \mathrm{~mm}$ superficiels du sédiment ont été conservés et fixés à l'alcool. Au laboratoire, $50 \mathrm{~cm}^{3}$ de ce sédiment ont été lavés sur un tamis de $62 \mathrm{~mm}$. Le résidu a ćtć tcinté au Rose Bengal (Walton, 1952), puis 
séché. Les foraminifères ont ensuite été séparés par flottage sur le tétrachlorure de carbone. Les comptages ont été effectués sous la loupe binoculaire. Le travail s'est décomposé en 3 étapes 1) le nombre total a été évalué, 2) 100 à 200 individus ont été comptés et déterminés en notant s'ils étaient vivants ou non, 3 ) le reste de la microfaune a été observé, sans comptage, pour noter les espèces présentes dans l'ensemble de l'échantillon. Les échantillons contenant moins d'une centaine d'individus n'ont pas été utilisés pour les comptages par espèce.

\section{Zone d'étude}

Le complexe lagunaire d'Iguape-Cananéia, au Sud de l'état de São Paulo est situé entre $24^{\circ} 40^{\prime}$ ' et $25^{\circ} 05^{\prime}$ 'de latitude sud et entre $47^{\circ} 25^{\prime}$ et $48^{\circ}$ de longitude ouest (Fig. 1). Il est isole de la mer par une île barrière d'environ $70 \mathrm{~km}$ de long (Ilha Comprida). En arrière de cette île, circule un chenal qui se divise en deux vers le sud (Mar de Cananéia et Mar de Cubatão) isolant a Ilha de Cananéia (Fig. 1). Les communications avec la mer se font par deux passes, aux deux extrémités de l'Ilha Comprida.

La zone d'ćtude se situe au Sud-Ouest de ce complexe: dans la Baía de Trapandé, au sud de la Mar de Cubatāo et au sud de la Mar de Cananéia. La mangrove qui peut être présente en divers points est particulièrement bien développée dans l'estuaire du Rio Itapitangui. On y rencontre essentiellement Rhizophora mangle, Avicennia schaueriana et Laguncularia racemosa, association décrite dans d'autres mangroves du Brésil mais qui atteint ici sa limite d'extension vers le sud.

Les eaux mixohalines de la lagune, sous la double influence des apports d'eau douce par les petits fleuves côtiers et des cycles de marée, subissent d'importantes variations journalières et saisonnières de salinité et de température. En règle générale, la salinité diminue quand la distance à la mer augmente, la stratification des eaux étant bien marquée, particulièrement dans la Baía de Trapandé (Miyao et al., 1986).

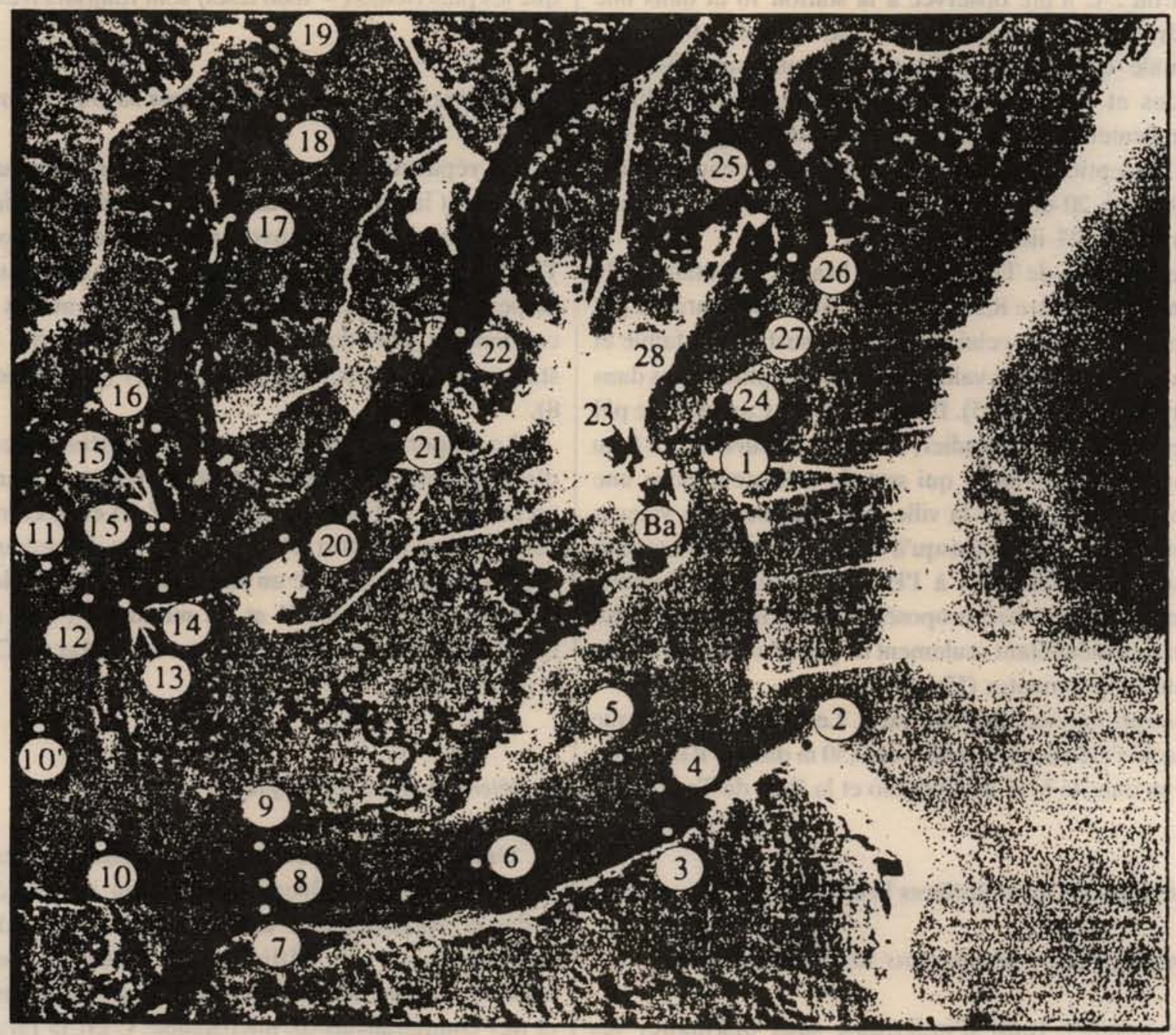

Fig. 2. Localisation des stations de prélèvement.

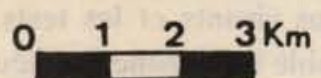


Les données concernant la teneur en oxygène dissous de l'eau montrent que le taux de saturation atteint 80 à 90\% dans la Mar de Cananéia, 85 à $100 \%$ dans la Baía de Trapandé et 75 à $80 \%$ dans la Mar de Cubatāo (Miyao et al., op. cit.)

La salinité est plus forte en hiver (période de pluviosité minimum) qu'en été, alors que le taux de nutrient est inférieur.

\section{Résultats}

\section{Caractéristiques hydrologiques pendant la période du} prélèvement

Au moment des prélèvement (octobre 1993), la température de l'eau variait entre $22,7^{\circ} \mathrm{C}$ près de l'embouchure ( $9 \mathrm{~h} 15)$ et $25,4^{\circ} \mathrm{C}$ dans le Rio Itapitingui (13h20). La température superficiclle est généralement supérieure de quelques dixièmes de degrés à la température du fond. Une différence anormalement forte d'environ $2^{\circ} \mathrm{C}$ a été observée à la station 16 et dans une moindre mesure à la station 15 (Fig. 3). Cette différence anormale correspond à l'apport d'eaux superficiclles chaudes et peu salées, comme le montre la salinité anormalement faiblc à ces mêmes stations (Fig. 4).

A l'exception des estuaires, la salinité des eaux de fond variait entre 20 et $25 \%$. La salinité de surface avait une valeur de 1 à $4 \%$ inférieure (Fig. 4).

Dans la Baía de Trapandé et le Mar de Cubatão, le pH des eaux varie entre 8,25 et 8,5 . La différence entre le $\mathrm{pH}$ des eaux de fond et celui des eaux de surface est faible et irrégulère (Fig. 5). Les valeurs sont un peu plus basses dans les estuaires (de 8 à 8,25). Dans la Mar de Cananéia, le pH est beaucoup plus irrégulier, avec des valeurs de 7 à 8 au voisinage de Rio Olaria qui sert de collecteur pour une partie des effluents de la ville de Cananéia. Des valeurs anormalement élevées (jusqu'à 9,3) ont été observées dans les eaux superficielles, à l'Est de Cananéia. Aucune explication n'a pu être proposée pour cette anomalie, les eaux concernées étant seulement un peu plus dessalées que les eaux environnantes (Fig. 4).

La transparence de l'eau varie. Les mesures au disque de Secchi s'étendent de moins de $0,50 \mathrm{~m}$ dans le Rio Olaria à $2,15 \mathrm{~m}$ dans le Mar de Cubatão et la Baía de Trapandé.

\section{Répartition des foraminiferes benthiques}

a) nombre de tests présents dans $50 \mathrm{~cm}^{3}$ de sédiment

Le nombre total d'individus, sans distinguer les individus vivants et les tests vides, est très variable sur l'ensemble de la zone étudiée. L'échantillon prélevé dans l'embouchure de la lagune (St. 2) ne contenait aucun test. Les échantillons les plus riches (St. 4, 14 et $\mathrm{Ba}$ ) en contenaient environ 3000 .

D'une manière générale, on constate que le nombre de tests est maximum (>1000), dans le chenal de la Baía de Trapandé (Fig. 6). On trouve également un grand nombre d'individus ( $>500$ ) dans l'entrée sud du Mar de Cananéia. Ensuite,cenombrediminueversleNord.

Le nombre d'individus vivants n'est pas proportionnel au nombre total d'individus (Fig. 7). Il peut en effet, y avoir un grand nombre de tests avec une faible proportion d'individus vivants. Dans la station 4, par exemple, il y a 6 $\%$ d'individus vivants pour un total de 3000 tests. Au contraire, la proportion d'individus vivants peut être forte dans des sédiments contenant peu de tests. Ceci se produit surtout dans les estuaires. La station 11, par exemple, ne contient que 21 individus, mais $70 \%$ d'entre eux sont vivants.

Il convient enfin de noter que, à l'exception de la station 2, située dans une zone de forts courants, les échantillons les plus pauvres sont constitués de sables grossiers alors que les plus riches ( $>1000$ tests) sont toujours très vaseux.

\section{b) Nombre d'espèces reconnues dans $50 \mathrm{~cm}^{3}$ sédiment}

La répartition du nombre total d'espèces suit assez fidèlement la répartition du nombre total d'individus. Les échantillons des plus pauvres sont ceux des stations 19 et 11 , les plus dessalées, qui ne contiennent que, respectivement, 2 et 5 espèces. Toutes les autres stations contiennent plus de 10 espèces, les plus riches étant la station 10 (32 espèces) et la station 27 (31 espèces) (Fig. 8).

Le rapport entre le nombre total d'espèces et le nombre d'espèces présentant des individus vivants est variable. Il est faible $(<1,5)$ dans les stations $10^{\prime}, 11$ et 16 et fort $(>6)$ dans les stations 10 et 24. La répartition des valeurs de ce rapport (Fig. 9) montre un maximum ( $>5$ ) dans le chenal de la Baía de Trapandé et un minimum $(<2)$ dans les estuaires et dans quelques stations de la Mar de Cananéia (1,23 et 26$)$.

\section{c) Déterminations spécifiques}

Une centaine d'espèces a été reconnue dans l'ensemble des échantillons (Tableau 1). Environ la moitié, parmi celles-ci appartiennent aux Rotaliina et une douzaine appartiennent aux Miliolina. L'étude systématique nécessite encore des recherches de descriptions originales et des comparaisons de microfaune. C'est la raison pour laquelle plusieurs espèces sont laissées en nomenclature ouverte. 


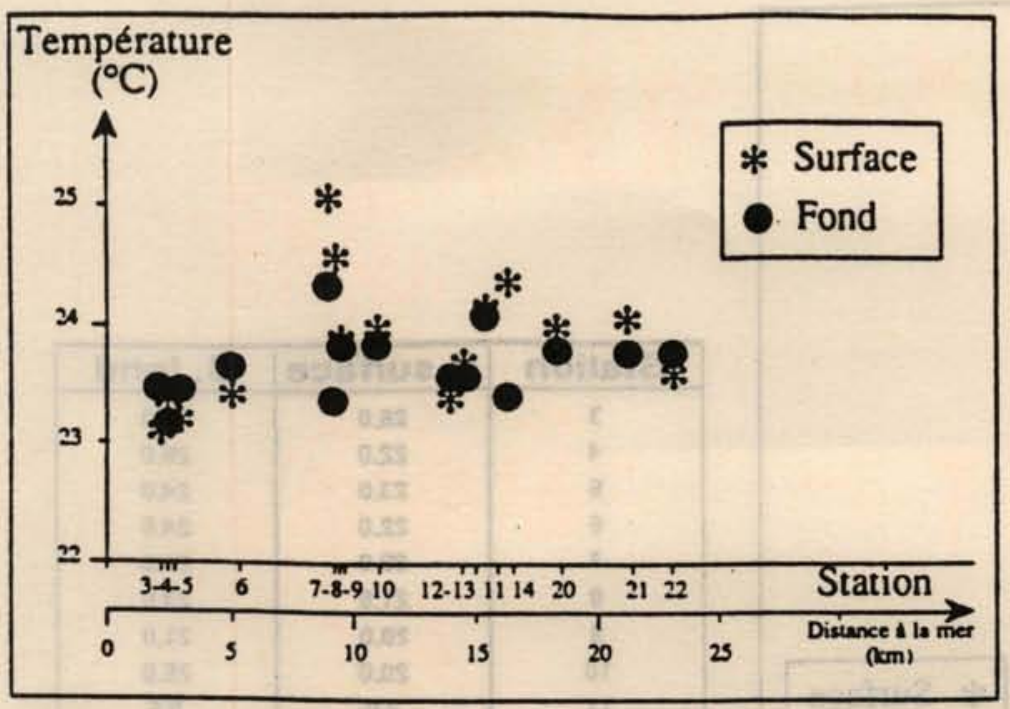

\begin{tabular}{|c|c|c|}
\hline Station & T.surface & T. fond \\
\hline 3 & 23.1 & 23.5 \\
4 & 23.4 & 23.1 \\
5 & 23.2 & 23.5 \\
6 & 23.4 & 23.7 \\
7 & 25.1 & 24.4 \\
8 & 24.6 & 23.3 \\
9 & 23.9 & 23.9 \\
10 & 24.0 & 23.9 \\
11 & 24.2 & 24.1 \\
12 & 23.7 & 23.5 \\
13 & 23.4 & 23.5 \\
14 & 24.4 & 23.4 \\
20 & 24.0 & 23.8 \\
21 & 24.1 & 23.8 \\
22 & 23.6 & 23.0 \\
\hline
\end{tabular}

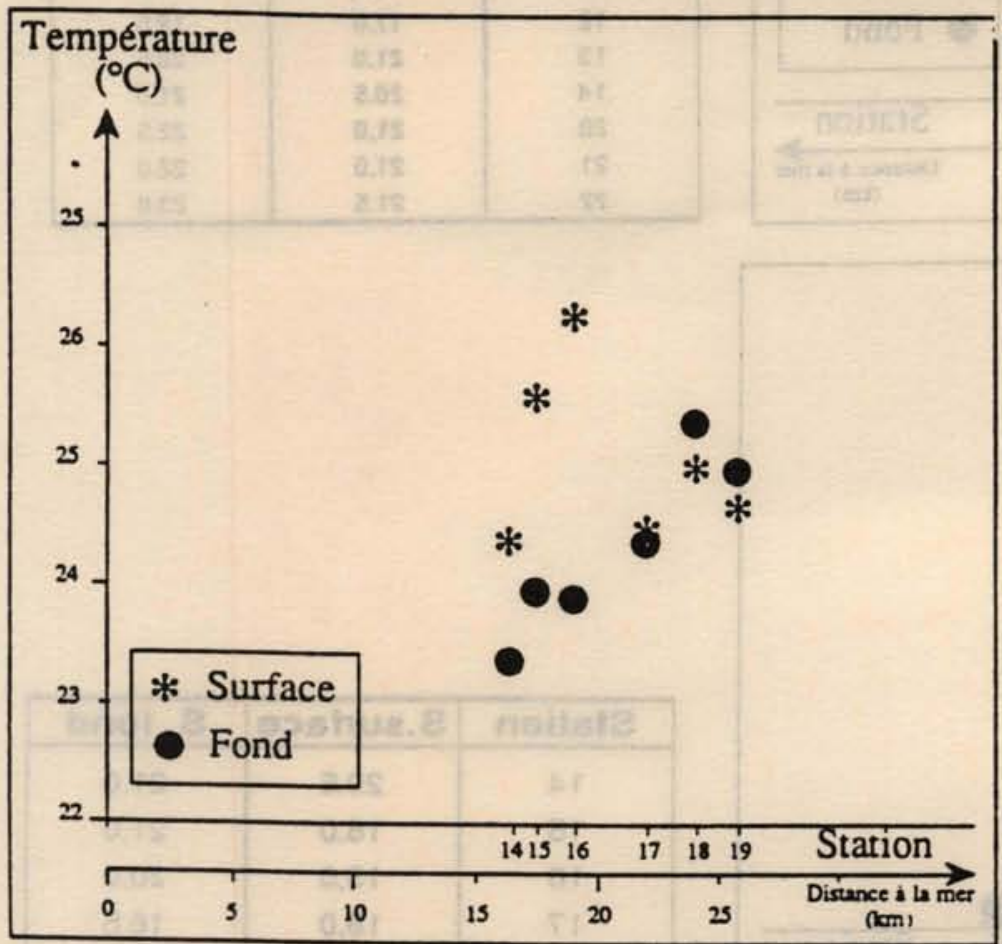

\begin{tabular}{|c|c|c|}
\hline Station & T.surface & T. fond \\
\hline 14 & 24.4 & 23.4 \\
15 & 25.6 & 24.0 \\
16 & 26.3 & 23.9 \\
17 & 24.5 & 24.4 \\
18 & 25.0 & 25.4 \\
19 & 24.7 & 25.0 \\
\hline
\end{tabular}

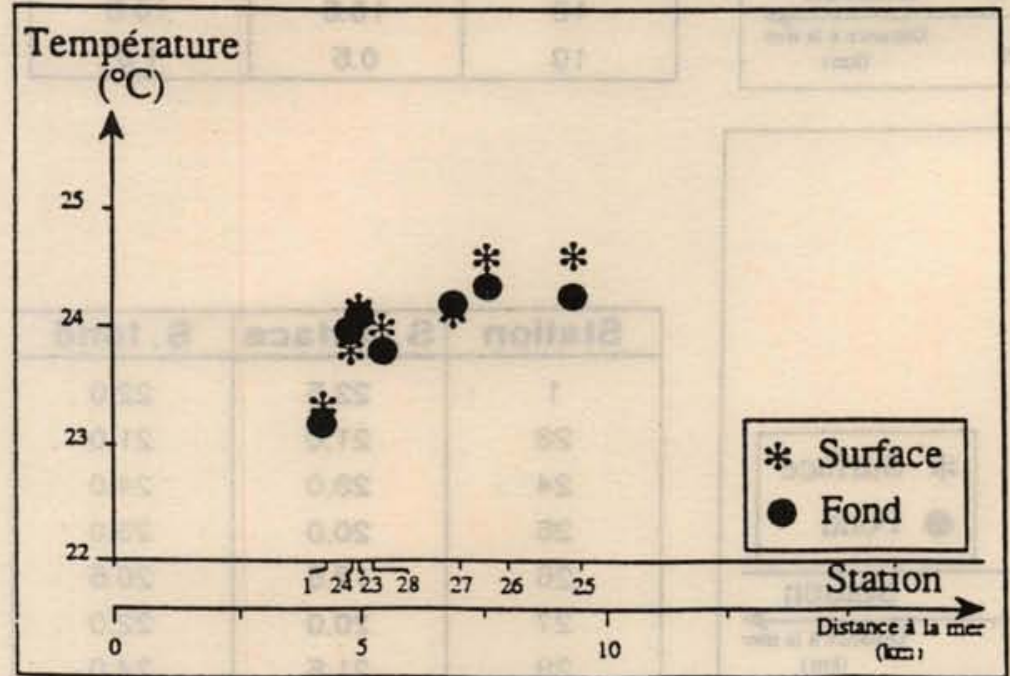

\begin{tabular}{|c|c|c|}
\hline Station & T.surface & T.fond \\
\hline 1 & 23.3 & 23.2 \\
23 & 24.1 & 24.1 \\
24 & 23.8 & 24.0 \\
25 & 24.6 & 24.3 \\
26 & 24.6 & 24.4 \\
27 & 24.1 & 24.2 \\
28 & 24.0 & 23.8 \\
\hline
\end{tabular}

Fig. 3. Température des eaux pendant la période d'échantillonnage. 


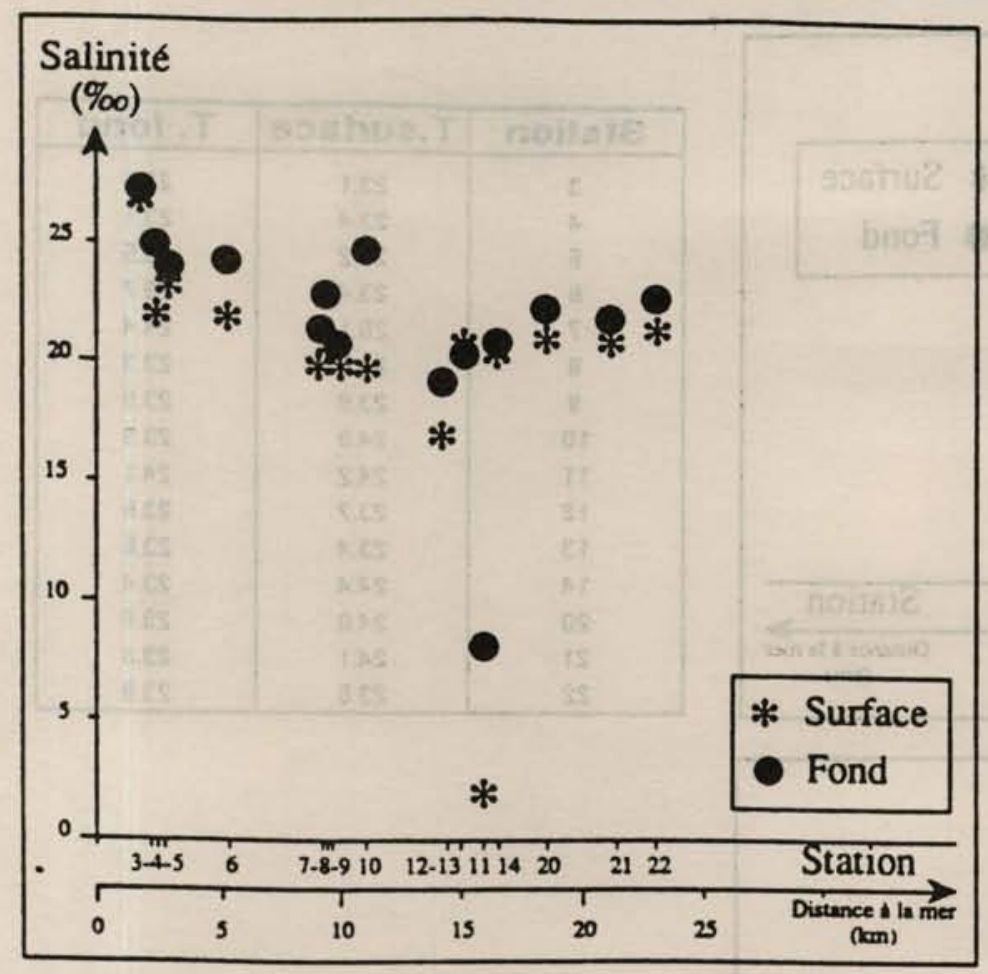

\begin{tabular}{|c|c|c|}
\hline Station & S.surface & S. fond \\
\hline 3 & 26,0 & 25.0 \\
4 & 22.0 & 25.0 \\
5 & 23.0 & 24.0 \\
8 & 22.0 & 24.5 \\
7 & 20,0 & 21.5 \\
8 & 21.5 & 23.0 \\
9 & 20.0 & 21.0 \\
10 & 20.0 & 25.0 \\
11 & 2.0 & 8.5 \\
12 & 17.0 & 19.5 \\
13 & 21.0 & 20.5 \\
14 & 20.5 & 21.0 \\
20 & 21.0 & 22.5 \\
21 & 21.0 & 22.0 \\
22 & 21.5 & 23.0 \\
\hline
\end{tabular}

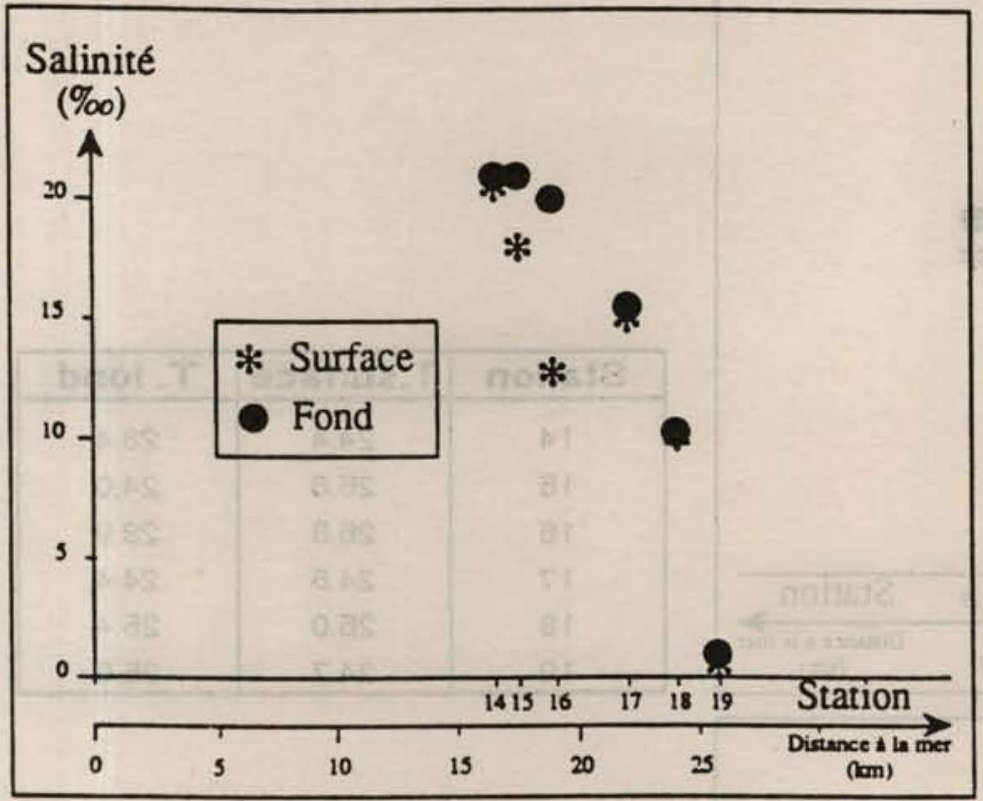

\begin{tabular}{|c|c|c|}
\hline Station & S.surface & S. fond \\
\hline 14 & 20.5 & 21.0 \\
16 & 18.0 & 21.0 \\
18 & 13.0 & 20.0 \\
17 & 15.0 & 16.5 \\
18 & 10.5 & 10.5 \\
19 & 0.5 & 1.0 \\
\hline
\end{tabular}

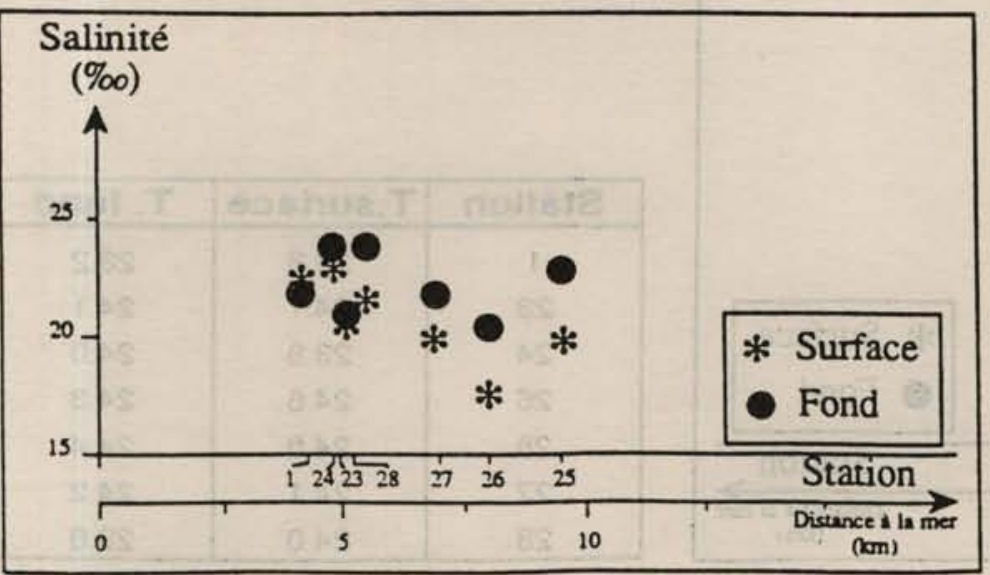

\begin{tabular}{|c|c|c|}
\hline Station & S.surface & S. fond \\
\hline 1 & 22.5 & 22.0 \\
23 & 21.0 & 21.0 \\
24 & 23.0 & 24.0 \\
25 & 20.0 & 23.0 \\
26 & 17.5 & 20.5 \\
27 & 20.0 & 22.0 \\
28 & 21.5 & 24.0 \\
\hline
\end{tabular}

Fig. 4. Salinité des eaux pendant la période d'échantillonnage. 


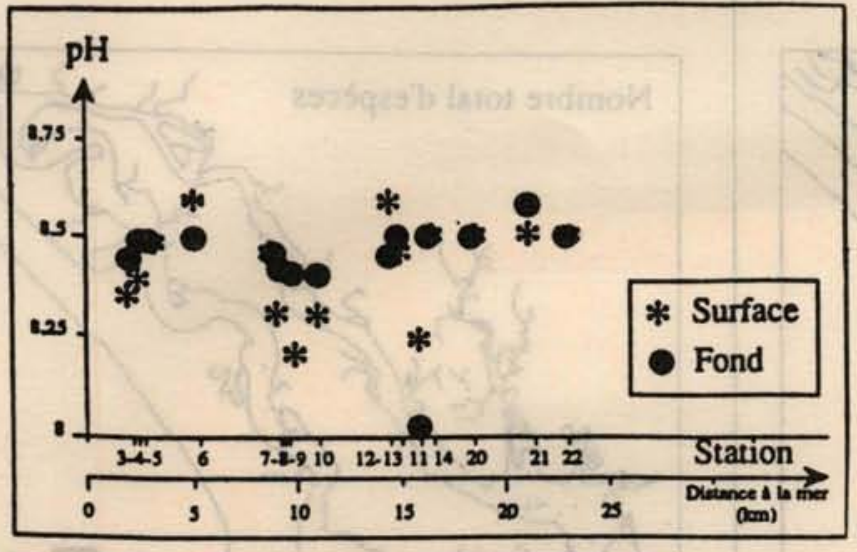

\begin{tabular}{|c|c|c|}
\hline Station & pH surt & pH lond \\
\hline 3 & 8.35 & 8.45 \\
1 & 8.40 & 8.50 \\
5 & 8.50 & 8.50 \\
8 & 0.50 & 8.80 \\
7 & 8.45 & 8.45 \\
8 & 8.30 & 8.41 \\
0 & 8.20 & 0.40 \\
10 & 8.30 & 8.40 \\
11 & 8.25 & 8.00 \\
12 & 6.60 & 8.45 \\
13 & 6.46 & 8.50 \\
14 & 8.50 & 8.50 \\
20 & 8.50 & 8.50 \\
21 & 0.50 & 0.60 \\
22 & 8.50 & 8.50 \\
\hline
\end{tabular}

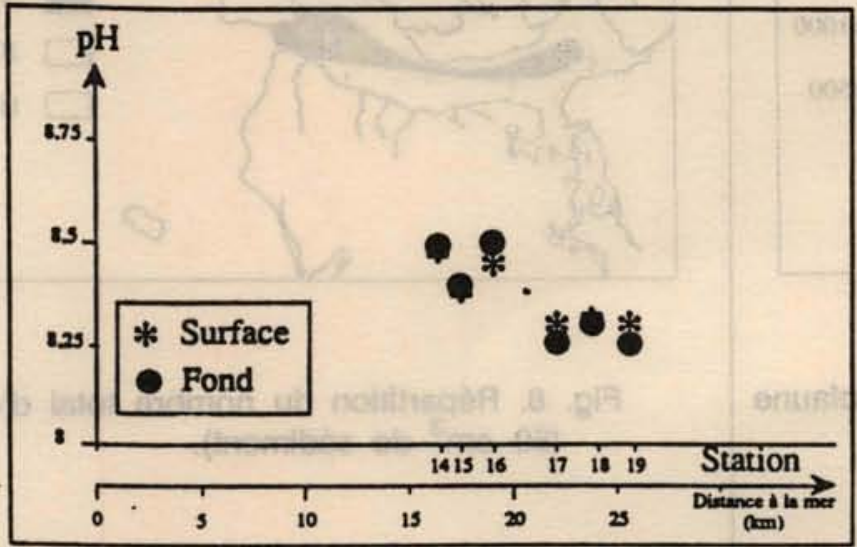

\begin{tabular}{|c|c|c|}
\hline Station & PH surf & PH fond \\
\hline 14 & 8.60 & 8.60 \\
15 & 8.40 & 8.40 \\
18 & 8.45 & 8.45 \\
17 & 8.30 & 8.25 \\
18 & 8.30 & 8.30 \\
19 & 8.30 & 8.25 \\
\hline
\end{tabular}

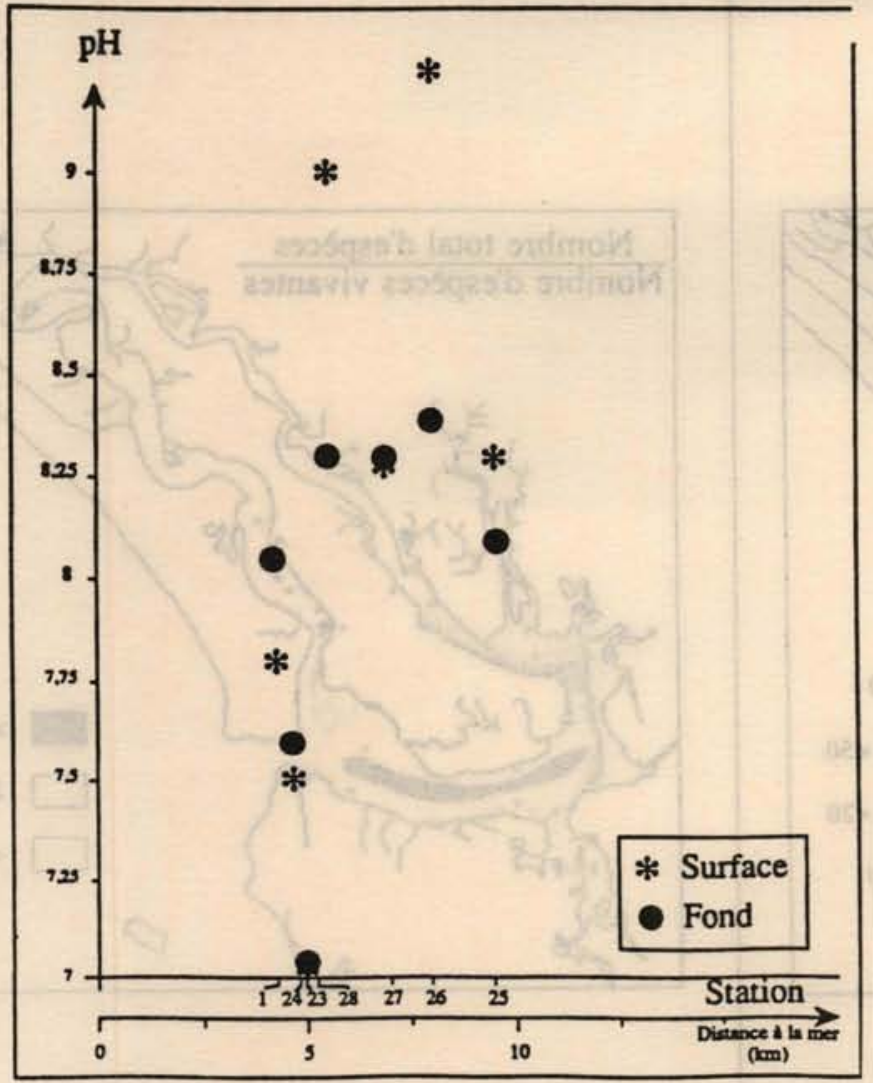

\begin{tabular}{|c|c|c|}
\hline Station & pH surt & pH fond \\
\hline 1 & 7.80 & 8.05 \\
23 & 7.00 & 7.00 \\
24 & 7.60 & 7.60 \\
25 & 8.30 & 8.11 \\
26 & 9.30 & 8.40 \\
27 & 8.30 & 8.30 \\
28 & 9.00 & 8.30 \\
\hline
\end{tabular}

Fig. 5. $\mathrm{pH}$ des eaux pendant la période d'échantillonnage. 


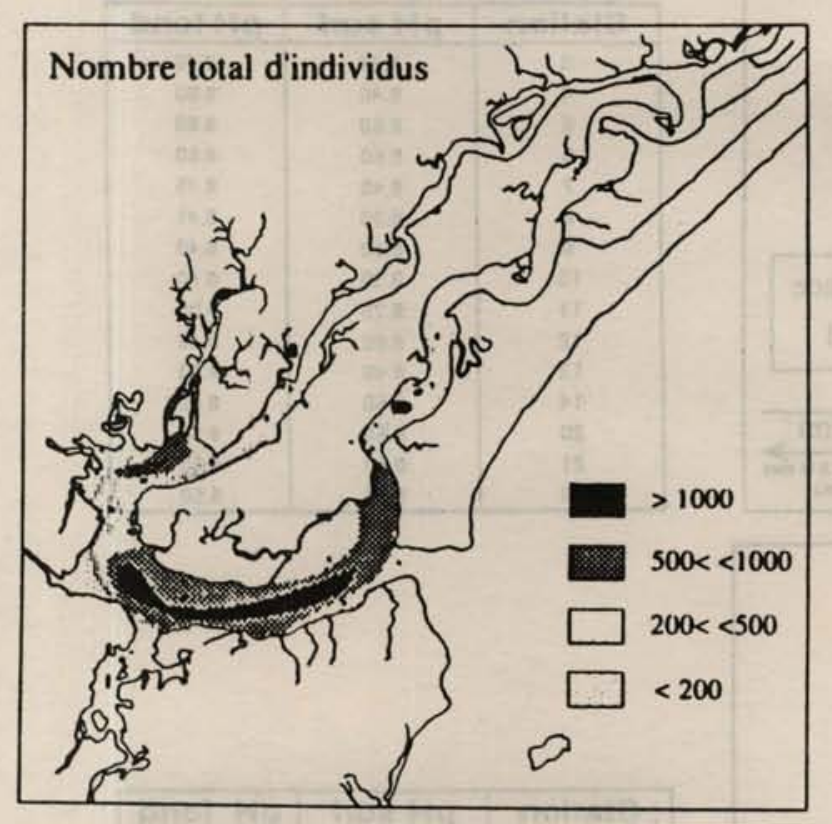

Fig. 6. Répartition quantitative de la microfaune totale $\left(50 \mathrm{~cm}^{3}\right.$ de sédiment).

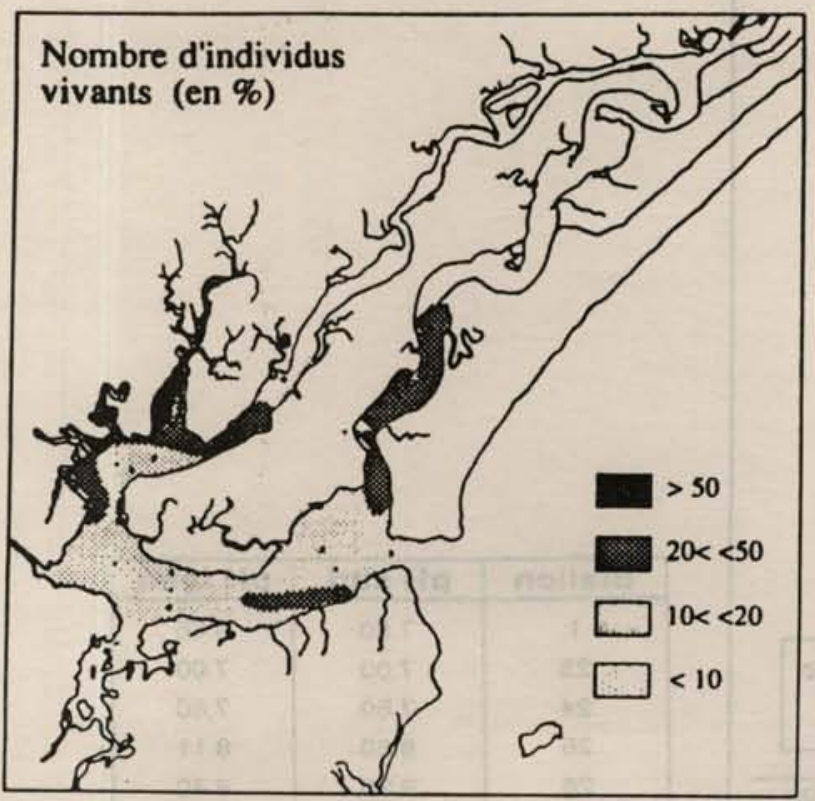

Fig. 7. Répartition quantitative des individus vivants (en pourcentage de la micrótaune totale).

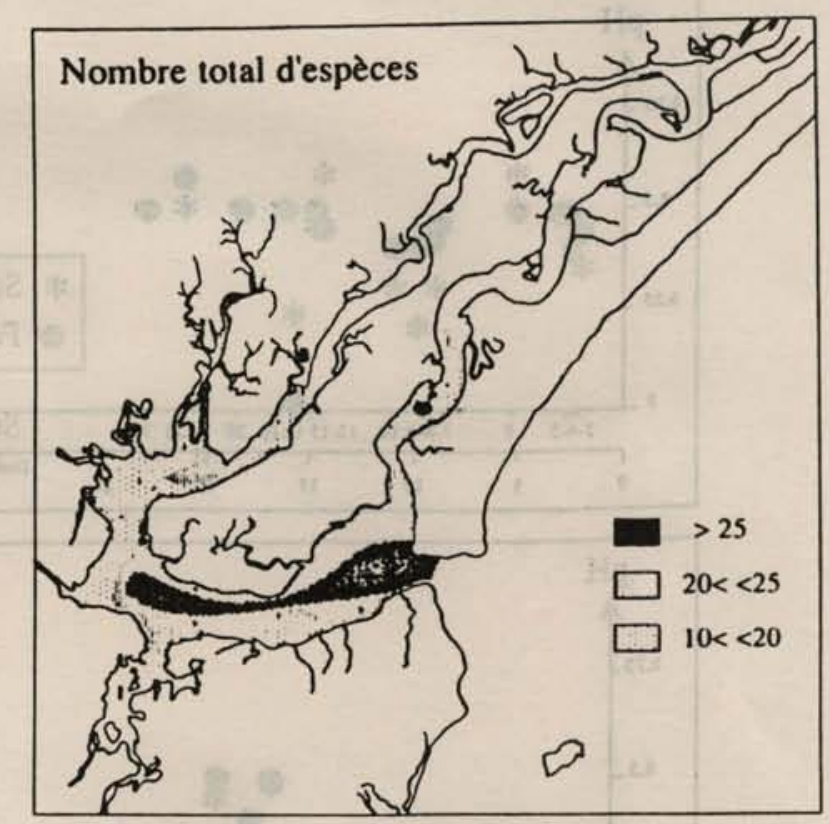

Flg. 8. Répartition du nombre total d'espèces (50 $\mathrm{cm}^{3}$ de sédiment).

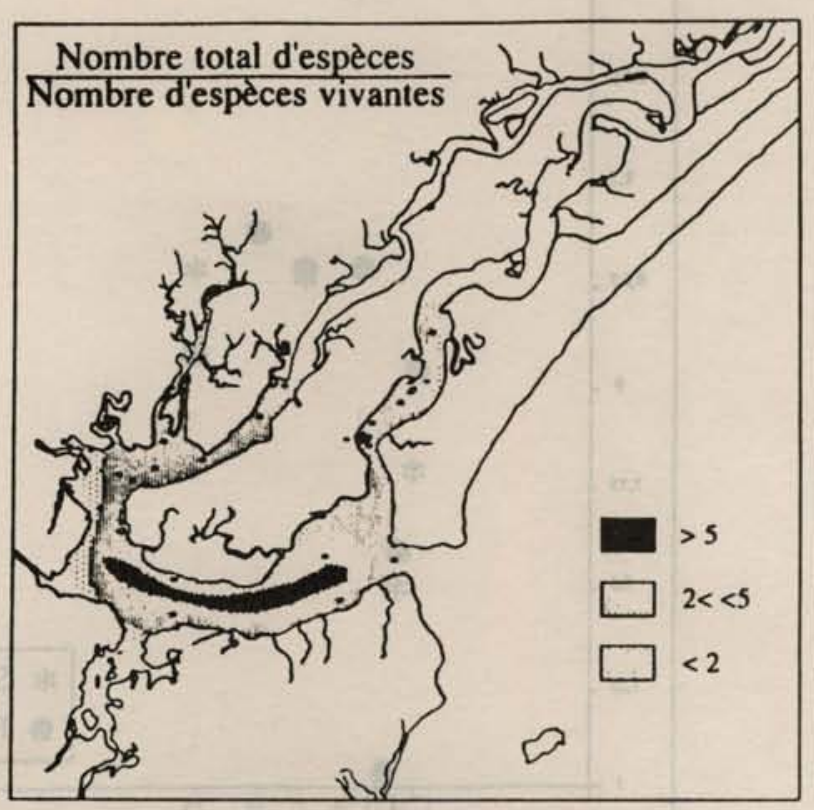

Fig. 9. Répartition du rapport nombre total d'espècies/nombre d'espèces vivantes. 
Tableau 1. Répartition des espèces de foraminifères récoliées dans le complexe laguno-estuarien d'Iguape-Cananéia

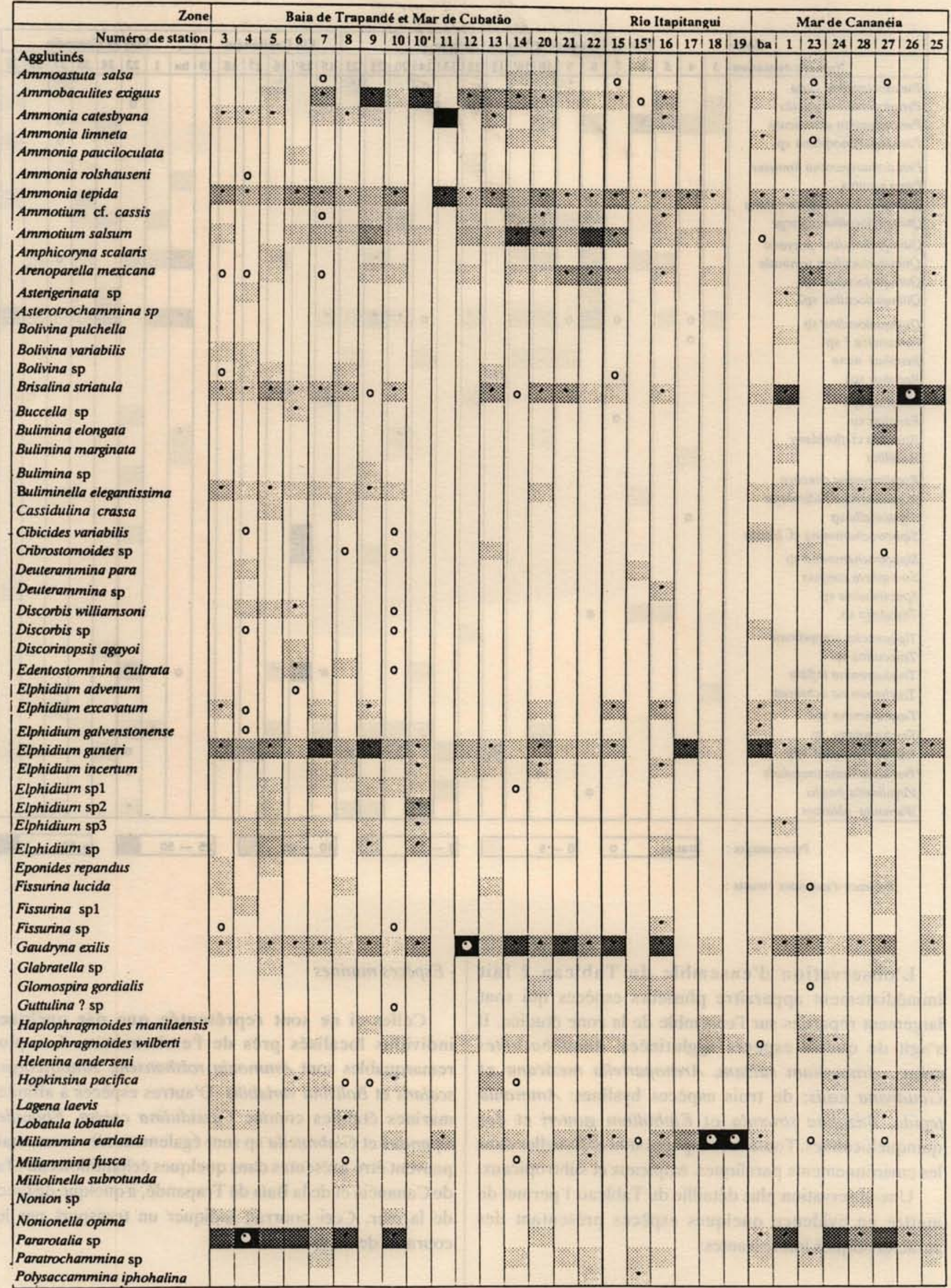


Tableau 1. Cont.

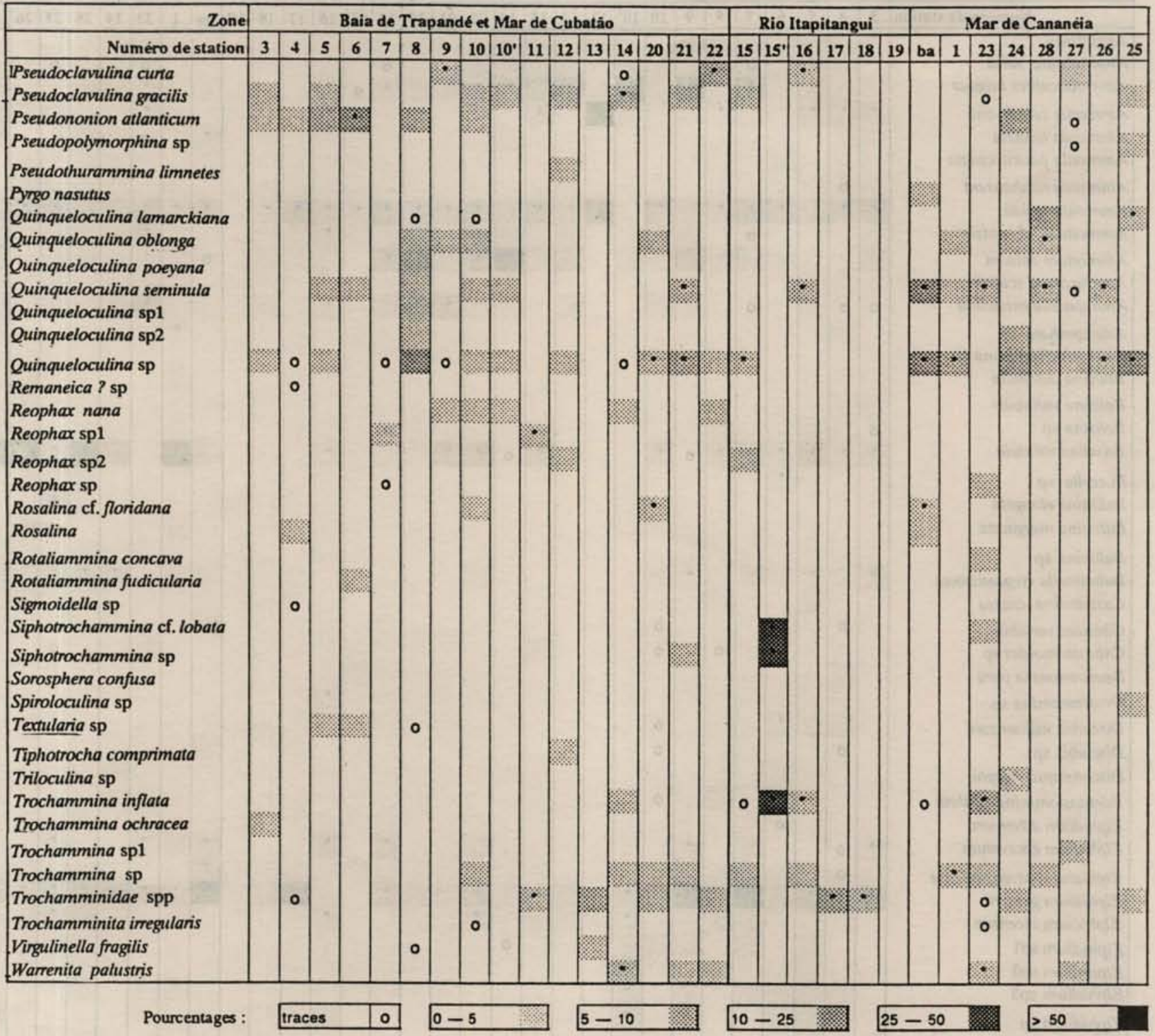

Presence d'individus vivants :

L'observation d'ensemble du Tableau 2 fait immédiatement apparaître plusieurs espèces qui sont largement réparties sur l'ensemble de la zone étudićc. Il s'agit de quatre espèces agglutinćes: Ammobaculites exigus, Ammotium salsum, Arenoparrella mexicana et Gaudryina exilis; de trois espèces hyalines: Ammonia tepida, Brizalina striatula et Elphidium gunteri et des quinqueloculines. Toutes ces espèces sont habituelles dans les environnements paraliques, tropicaux et subtropicaux.

Une observation plus détaillic du Tablcau 1 permet de mettre en évidence quelques espèces présentant des caractéristiques intéressantes:

\section{- Espèces marines}

Celles-ci ne sont représentée que par quelques individus localisés près de l'embouchure. Les plus remarquables sont Ammonia rolshauseni, Amphycoryna scalaris et Bolivina variabilis. D'autres espèces à affinités marines établies comme Cassidulina crassa, Eponides repandus et Glabratella sp sont ćgalement très rares, mais peuvent être présentes dans quelques échantillons du Mar de Cananćia et de la Baía de Trapandé, à quelque distance de la mer. Ceci pourrait indiquer un transport par les courants de marées. 
- Espèces à affinités marines, tolérant un confinement modéré

Deux espèces illustrent bien ce comportement. Il s'agit de Pseudononion atlanticum qui pénètre dans toute la Baía de Trapandé et se retrouve dans 2 échantillons du Mar de Cananéia et de Pararotalia sp. Abondante dans la Baía de Trapandé et dans la Mar de Cananćia, cette dernière espèce a son abondance relative qui décroît quand la distance à la mer augmente (Fig. 10).

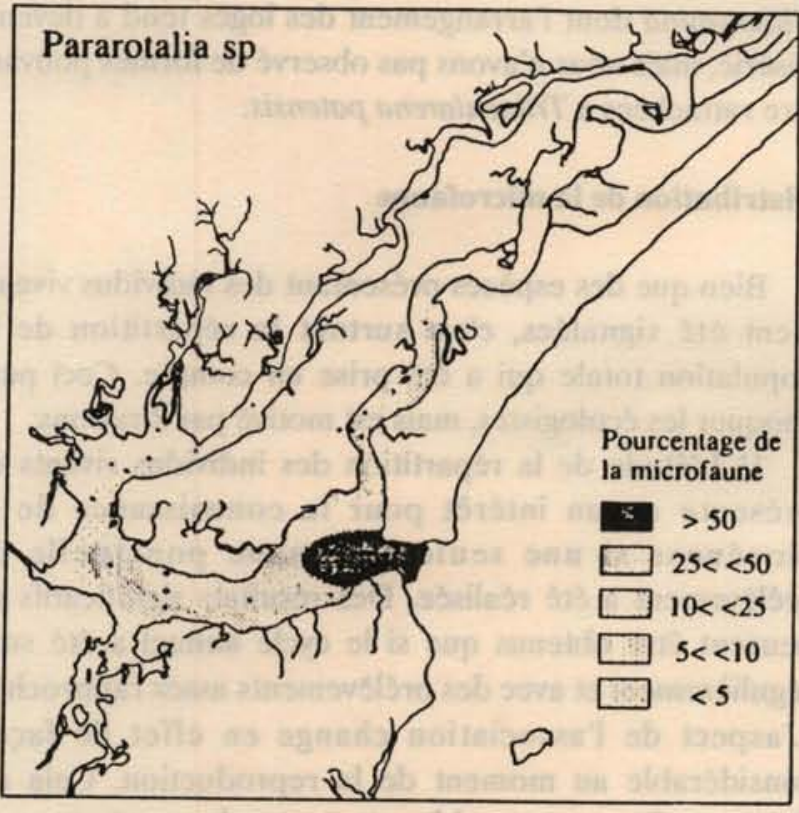

Fig. 10. Répartition quantitative de Pararotalia sp.

\section{- Espèces d'environnements confinés}

A l'inverse des espèces précédentes, certaines espèces ont leur abondance relative qui augmente avec la distance à la mer et donc, le confinement. Il s'agit en particulier de Ammotium salsum, Gaudryina exilis et Miliammina earlandi. Cette dernière espèce est la plus résistante au confinement dans les eaux dessalées et c'est elle qui prédomine dans le Rio Itapitangui.

Deux espèces présentent également des caractéristiques intéressantes. Ce sont Arenoparrella mexicana et Haplophragmoïdes wilberti. Ces espèces, qui ont montré en Afrique leur affinité pour les zones à matière organique abondante (Debenay, 1990) sont présentes dans le Rio Olaria, où elles sont toutes deux vivantes (st. 23) et à proximité de l'embouchure de ce fleuve. Elles sont également présentes dans les stations 21 et 22 où $A$. mexicana est vivante. Ces deux zones correspondent aux emplacements des rejets des effluents d'égoûts de la ville de Cananéia.

\section{- Espèces épiphytes}

Le prélèvement par grattage d'un échantillon d'algues sur les racines immergées d'un palćtuvier (st. 15') a permis de mettre en évidence une microfaune épiphyte constituée essentiellement de Trochammina inflata, Siphotrochammina lobata et Siphotrochammina sp.

\section{- Cas particulier}

La répartition de Brizalina striatula est remarquable. Cette espèce est en effet largement répartie dans toute la zone étudiée, mais son abondance relative est la plus forte dans la Mar de Cananéia. Aucune explication ne peut être proposée, pour le moment, concernant cette répartition.

\section{Discussion}

\section{Populations et taxonomie}

La microfaune observée dans le complexe laguno-estuarien d'Iguape-Cananéia présente de nombreux points communs avec celles qui ont été décrites dans d'autres environnements laguno- estuarien du Brésil, sur la côte sud (Madeira Falceta, 1974) et dans la Baía de Sepetiba (Zaninetti et al., 1977). Elle s'en distingue cependant par sa plus grande richesse, puisqu'une centaine d'espèces ont été inventoriées contre une cinquantaine dans la Lagoa dos Patos, une soixantaine dans la Baía de Sepetiba et 27 seulement dans la Baía de Paranaguá, la plus proche de notre zone d'étude.

Cette plus grande richesse pourrait résulter de l'équilibre qui existe ici entre l'influence continentale et l'influence marine. Celle-ci, en raison de la large ouverture de la Baía de Trapandé sur la mer, se fait sentir jusque dans les marais et mangroves peuplant les estuaires. La salinité de fond ne varie qu'entre $27 \%$ et $28 \%$ au cours d'un cycle de marée dans la Mar de Cubatāo et de $29 \%$ à $34 \%$ à l'entréc du Mar de Cananéia, en hiver (Miyao et al., 1986). A l'embouchure de la Lagoa dos Patos, au contraire, Closs (1963) rapporte des salinités pouvant varier de 0 à $30 \%$, ce qui explique la faible quantité d'espèces à affinités marines observée dans cette lagune.

Les associations d'espèces typiques des environnements confinés sont très comparables dans les différents environnements paraliques de la région. Les différences observées peuvent être dues en partie à des 
distorsions dans la détermination des espèces, résultant d'une interprétation différente des descriptions publiées dans la littérature ou d'une différence d'appréciation des caractéristiques morphologiques. Bien que cette étude n'ait pas un objectif systématique, il est possible de citer quelques cas oú une telle distorsion est possible.

Dans la Lagoa dos Patos, Closs signale la présence de Textularia earlandi, également signalée par Scott et al. (1990) dans la lagune de Garatuba. Il sera nécessaire d'observer les échantillons issus de ces environnements pour le vérifier, mais il est fort probable qu'il s'agisse en fait de Gaudryina exilis que nous avons trouvée à Cananéia et qui a été signalée dans la Baía de Sepetiba. Cette espèce présente un stade trisérié très court, difficilement observable, ce qui rend la confusion aisée.

Un autre problème systématiquement délicat concerne Ammonia, genre habituellement très fréquent dans les environnements paraliques. A l'exception de $A$. rolshauseni, très typique et à affinité marine que nous avons trouvée en petit nombre, toutes les autres formes posent des problèmes systématiques. Sclon Petri (1971), toutes les formes vivant sur les côtes du Brésil sont à rattacher à $A$. catesbyana. La définition originale de d'Orbigny indique que cette espèce possède 10 loges dans son dernier tour de spire. Ce n'est pas le cas de tous les individus que nous avons observés. Bien que de nombreuses formes intermédiaires existent, il nous a semblé possible de distinguer 2 espèces: $A$. tepida et $A$. catesbyana. Les formes à loges plus nombreuses (8à 10) et à contour peu à pas lobé ont été rattachées à Ammonia catesbyana. Leur morphologie rappelle celle d'Ammonia parkinsoniana, à l'exception du bouton central, bien développé chez. $A$. parkinsoniana. Malgré l'incertitude qui peut planer sur ces déterminations, il n'est pas utile de tenter de différencier ces espèces (qui pourraient être des morphotypes) puisque leur comportement vis à vis des conditions d'environnement peut être différent. Ainsi, en Afrique, par exemple, Ammonia tepida et $A$. parkinsoniana réagissent différemment à la salinité de l'eau (Debenay et al., 1993).

Ammoastuta inepta a été reconnue comme synonyme de $A$. salsa par Saunders (1958). Toutefois, Brönnimann (1986) considère que ces 2 espèces qui vivent dans des environnements différents, doivent être distinguées. $A$. salsa est l'espèce caractéristique des environnements paraliques. C'est donc l'espèce que nous retiendrons ici.

Pseudothuramnina limnetes est décrite comme une espèce généralement libre, mais pouvant parfois se fixer. Tenant compte de cette possibilité de fixation, nous désignons sous le nom de $P$. limnetes des formes fixées qui présentent des caractéristiques qui pourraient les rapprocher d'Ammolagena clavata. Toutefois, le genre Ammolagena est caractéristique d'environnements marins relativement profonds. Notons qu'il existc une certaine confusion dans la nomenclature des formes agglutinées uniloculaires fixées.

Les Miliammina que nous avons observées dans les environnements les plus confinés et les plus dessalés sont de couleur brune et possèdent une dent simple, bien développée, caractéristique de $M$. earlandi. Les Miliammina se comportent donc différemment dans le complexe laguno-estuarien de Iguape-Cananéia et dans la Lagoa dos Patos, où c'est $M$. fusca qui occupe le même type d'environnements (Closs, 1963). Cette dernière espèce est ćgalement présente à Cananéia, mais en moins grand nombre que $M$. earlandi. Nous avons observé quelques Miliammina dont l'arrangement des loges tend à devenir trisérié, mais nous n'avons pas observé de formes pouvant être rattachées à Trilocularena patensis.

\section{Distribution de la microfaune}

Bien que des espèces présentant des individus vivants aient été signalées, c'est surtout la répartition de la population totale qui a été prise en compte. Ceci peut choquer les écologistes, mais est motivé par 2 raisons:

1) L'étude de la répartition des individus vivants ne présente aucun intérêt pour la connaissance de la biocénose si une seule campagne ponctuelle de prélèvement a été réalisée. Des résultats significatifs ne peuvent être obtenus que si le cycle annuel a été suivi régulièrement et avec des prélèvements assez. rapprochés. L'aspect de l'association change en effet de façon considérable au moment de la reproduction. Cela est d'autant plus remarquable que toutes les espèces ne se reproduisent pas en même temps et que la période de reproduction d'une espèce peut changer suivant son milieu de vie à l'intérieur d'une même lagune comme l'ont montré Closs \& Madeira (1968) dans la Lagoa dos Patos.

2) Le but de cette étude est avant tout écologique et paléoécologique. La présence de tests marins, même s'ils ont été transportés post mortem par les courants de marée indiquent une influence marine. Ils constituent donc un indice écologique important. Cet indice est d'autant plus important que c'est cette association de tests morts qui sera fossilisée et qui sera utilisée pour des interprétations palćoécologiques. De nombreux auteurs ont déjà montré qu'il ćtait bien préférable dans ce cas de travailler sur la population totale sans se préoccuper des individus vivants et parmi eux Akpati (1975); Scott \& Medioli, (1980); Beurlen \& Hilterman (1983).

Il n'est évidemment pas dans notre intention de nier l'intérêt de l'ćtude des biocénoses. C'est un type d'étude tout à fait indispensable pour comprendre le fonctionnement détaillé des écosystèmes, à condition que ces études soient réalisćes dans de bonnes conditions. La question qui pourra se poser alors est de savoir comment se fait lc passage de la biocénose à la thanatocénose. 
La distribution des foraminifères dans la zone étudiće est caractérisée par l'évolution depuis des microfaunes à affinités marines, dominées par les espèces calcaires près de l'embouchure, vers des faunes où les espèces agglutinées prennent de plus en plus d'importance. Ces faunes s'enrichissent en particulier en Ammotium salsum et Gaudryina exilis pour aboutir enfin, dans les zones les plus confinées et à salinité três faible, à des associations oligospécifiques oú domine Miliammina earlandi.

Ce type d'évolution est habituel dans tous les environnements paraliques. C'est en particulier celui qui a été décrit par Zaninetti et al. (1977) dans la Baía de Sepetiba et par Closs dans la Lagoa dos Patos. Cet auteur met en évidence 5 associations de foraminifères regroupées selon 5 zones qui sont, de la mer vers le fond de la lagune: la zone pré-marine, la zone sub-marine, la zone pré-mixohaline, la zone mixohaline et la zone pré-limnique. Dans la zone pré-marine, dominent les formes calcaires avec en particulier, Elphidium discoidale, Ammonia beccarii, Quinqueloculina seminula auxquelles s'ajoutent Buccella peruviana campsi, Buliminella elegantissima, Brizalina striatula, Poroeponides lateralis, Bucella frigida, Bulimina marginata et Discorbis williamsoni. Ces espèces ont des affinités marines, mais toutes ont également été signalées dans des environnements de Baías, d'estuaires ou de lagunes. Vers l'intérieur de la lagune, d'autres espèces calcaires voient leur abondance relative croître. Ce sont Elphidium gunteri et $E$. excavatum. Toutefois, l'évolution la plus remarquable est l'apparition et le développement de plus en plus important des espèces agglutinées avec Ammotium salsum, Ammotium cassis, Textularia earlandi, Haplophragmoides wilberti, Trilocularena patensis, Arenoparrella mexicana et Miliammina fusca. Ce sont ces deux dernières espèces qui résisteront au confinement jusqu'en zone pré-limnique avec dominance de $M$. fusca.

Nous avons calculé l'indice de diversité $\alpha$ de Fischer, en utilisant l'abaque de Murray (1973). Les valeurs sont comprises entre 2 et 8 dans l'ensemble de la zone. La répartition de ces valeurs est parfaitement alćatoire à l'intérieur de la lagune. Ceci confirme que l'indice $\alpha$ n'est pas adéquate pour déterminer les variations des conditions environnementales à l'intérieur d'un système paralique, comme cela a été noté auparavant par Zaninetti et al. (1977) et par Debenay (1990).

La répartition de Pararotalia, abondant dans la Mar de Cananéia montre que celle-ci est moins confinée que le Mar de Cubatāo ou cette espèce est absente (Fig. 10).

\section{Transport et dépôt post mortem des tests}

Un prélèvement a été cffectué sur les racines d'un Rhizophora (St. 15'). Il est très riche en Trochammina et
Siphotrochammina. Or l'échantillon 15, prélevé à peu de distance, mais dans le chenal, ne contenait que très peu de ces tests, de même que les autres échantillons situés à proximité. Il semble donc qu'après leur mort, ces tests soient peu déplacés. Une étude plus détaillée sera nécessaire pour déterminer l'étendue de la dispersion post mortem de cette espèce.

Nous avons montré que la plupart des échantillons contenant un grand nombre d'espèces se trouvent dans l'axe de la Baía de Trapandé. Parmi ceux ci, les plus riches sont les échantillons 8 ( 28 espèces) et 10 ( 32 espèces).

Il se trouve que ce sont aussi 2 des stations parmi celles qui ont le plus faible pourcentage en individus vivants. Ces deux caractéristiques semblent indiquer que ces stations sont plus des lieux d'accumulation après transport post mortem que des lieux favorables à la vie des foraminifères. Ceci semble confirmé par la présence de tests d'origine marine. Cette accumulation pourrait résulter d'un changement de régime de courant, dû au déboucher du canal d'Ararapira, qui provoquerait le dépôt des tests en suspension. Il ne s'agit là que d'une hypothèse qui devra être vérifiée.

\section{Influence de la pollution}

Bien que des analyses d'eaux n'aient pas été faites dans le Rio Olaria, il est de notoriété publique qu'une partie des égouts de la ville y aboutit. L'aspect des eaux et l'odeur des sédiments nous ont permis de nous en persuader. A titre d'étude préliminaire, nous avons prélevé un échantillon dans ce fleuve afin d'apprécier l'impact de ce type de pollution sur la microfaune. Il apparaît en définitive que celle-ci est relativement riche avec 600 individus pour $50 \mathrm{~cm}^{3}$, dont $20 \%$ sont vivants et avec 28 espèces représentées, dont 15 vivantes. C'est l'un des nombres les plus élevés. Il semble donc qu'à l'endroit où le prélèvement a été effectué, la pollution soit plus favorable que néfaste à la microfaune. Ceci pourrait être dû au type de pollution, essentiellement organique qui règne dans le fleuve, associé à un renouvellement régulier de l'eau à chaque marée. Le seul indice, dans la microfaune, de l'abondance de matière organique est la présence d'une forte proportion d'Arenoparella mexicana associée à Haplophragmoides wilberti, tous deux présentant des individus vivants. Il n'apparaît pas de déformation massive des tests comme cela est de règle dans les zones très pollućes (Schafer \& Sen Gupta, 1969; Alve, 1991; Sharifi et al., 1991).

Des études plus détaillées, portant sur un plus grand nombre d'échantillons sont à envisager dans le Rio Olaria et au déboucher de l'égout principal, dans la Mar de Cubatâo. 
Comparaison avec les microfaunes des environnements paraliques d'Afrique de l'Ouest

Les microfaunes des environnements paraliques Ouest-Africains et Brésiliens présentent de grandes affinités, comme cela a déjà été signalć (Debenay, 1990). Les différences essentielles sont: la présence au Brésil de quelques espèces non signalées en Afrique (Ammotium cassis, Ammolagena clavata, Pseudoclavulina curta, Deuterammina spp, Haplophragmoides manilaensis, Rotaliammina spp, Sorosphaera confusa, Trochamminita irregularis et Warrenita palustris) et la présence en Afrique de quelques espèces non signalées au Brésil dont, en particulier, Eggerelloides scabrum.

\section{Conclusion}

Cette étude s'inscrit dans l'ensemble des études des microfaunes des environnements paraliques du Brésil. Elle permet de décrire la microfaune présente dans le complexe laguno-estuarien d'Iguape-Cananéia en faisant apparaître la richesse de cet environnement qui fournit deux fois plus d'espèces que les environnements décrits jusqu'alors, avec de nombreuses espèces à test carbonaté.

Les principales caractéristiques qui ressortent de l'analyse de la microfaune sont:

- une influence marine qui pénètre profondément dans la lagune;

- un confinement qui augmente progressivement avec la distance à la mer et qui ne devient maximum que dans les estuaires des petites rivières qui se jettent dans la lagune;

- une différence de comportement entre le Mar de Cubatāo et le Mar de Cananéia, cette dernière étant moins confinée;

- un faible impact de la pollution qui ne se traduit que par le développement de 2 espèces favorisces par l'augmentation de la teneur en matières organiques (Arenoparrella mexicana et Haplophragmoides wilberti) mais qui n'engendre pas de deformation massive des tests comme cela est le cas dans les environnements très pollués.

Des études plus détaillées des biocénoses permettront de mieux comprendre le fonctionnement global du système et de définir, entre autre, l'impact exact de la pollution en vue d'appliquer ces connaissances à la compréhension de zones où la pollution est beaucoup plus forte.

\section{Remerciements}

Les auteurs remercient la FAPESP (Fundação de Amparo à Pesquisa do Estado de São Paulo), qui a permis la préparation et l'aboutissement de ce projet. Ils remercient également le Muséum d'Histoire Naturelle de Genève, et en particulier D. Decrouez et C. Besnier qui ont grandement facilité la recherche bibliographique en permettant l'accès à la Bibliothèque de P. Brönnimann.

\section{References}

AKPATI, B.N. 1975. Foraminiferal distribution and environmental variables in Eastern Long Island Sound, New York. J. foram Res. 5(2):127-144.

ALVE, E. 1991. Benthic foraminifera in sediment cores reflecting heavy metal pollution in Sorfjord, Western Norway. Washington. J. foram Res., 21(1):1-19.

BEURLEN, G \& HILTERMANN, H. 1983. As biocenoses do mangue de Guaratiba, Rio de Janeiro, Brasil. B. Petrobrás, Rio de Janeiro, 26(4):259-267.

BRÖNNIMANN, P. 1978. Recent benthonic foraminifera from Brasil-Morphology and Ecology: Part 3. Notes on Asterotrochammina Bermuda and Seiglie. Notes Lab. palaeont., Université de Genève, 3:1-8.

1979. Recent benthonic foraminifera from Brazil - Morphology and Ecology. Part 4. Trochaminids from the Campos shelf with description of Paratrochammina n. gen. Paläont. Z., Stuttgart, 63:5-25.

1980. Recent benthonic foraminifera from Brasil - Morphology and Ecology. Part 5. Primétéve agglutinated foraminifera from the Campos and Bahia shelf. Paläont. Z., Stuttgart. 63:67-89.

1986. On morphology and taxonomy of Amoastuta Cushman \& Brönnimann, 1948 (Protista: Foraminiferida). Paläont. Z, Stuttgart, $60(1 / 2): 29-44$.

\& BEURLEN, G. 1977a. Recent benthonic foraminifera from Brasil - Morphology and Ecology. Part 1. Polystomammininae, new subfamily. The Trochamminidae and description of Polystomamminina planulata (Mikhale Vitali), from the Campos shelf. Archs Sci. Genève, 30:77-90. 
BRÖNNIMANN, P. \& BEURLEN, G. 1977b. Recent benthonic foraminifera from Brasil - Morphology and Ecology. Part 2 \& 3. Cribrostomoides Cushman and Haplophragmoides Cushman from the Campos shelf. 4. Trochammina brasiliensis Brönnimann and Beurlen, n.sp. from the Campos shelf. Archs Sci. Genève., 30:243-262.

\& DIAS-BRITO, D. 1982. New Lituolacea (Protista, Foraminiferida) from shallow waters of the Brazilian shelf. J. foram. Res., 12:13-23.

; MOURA, J. A. \& DIAS-BRITO, D. 1981. Estudos ecológicos na Baía de Sepetiba, Rio de Janeiro, Brasil: Foraminíferos. In: Congresso Latino Americano de Paleontologia, 2., Porto Alegre, 1981. Anais. Porto Alegre. p.862-875.

CARMOUZE, J.-P.; GIANESELLA-GALVĀO, S. M. F. G.; AIDAR ARAGÃO, E.; NISHIHARA, L.; SARTI, C. C.; ABE, D. S. \& MESOUITA, H. de S. L. 1986. Interaçōes biogeoquímicas e produçāo primária na interface do complexo estuarino lagunar de Cananéia com o seu manguezal. Tech. Rep., FAPESP, São Paulo, 63 p.

CLOSS, D. 1963. Foraminíferos e tecamebas de Lagoa dos Patos (RS). Bol. Esc. Geol., Porto Alegre, 11:1-130.

1964. Ecological distribution of Foraminifera and Thecamoebiina in the Patos lagoon. Southern Brazil. Arch. Oceanogr. Limnol., Venise, 13(2):297-302 .

\& MADEIRA, M. L. 1962. Tecamebas e foraminíferos do Arroio Chuí (Santa Vitória do Palmar, R. Grande do Sul, Brasil). Iheringia, Zool., Porto Alegre, 19:1-44.

\& 1966. Foraminifera from the Paranaguá Bay, State of Paraná. Curitiba. Bol. Univ. Fed. Paraná, Zoologia. 10:139-162.

$$
\text { \& }
$$

1967. Foraminíferos e tecamcebas aglutinantes da Lagoa de Tramandaí, no Rio Grande do Sul. Iheringia, Zool., Porto Alegre, 35:7-31.

\& 1968. Seasonal variations of brackish Foraminifera in the Patos lagoon Southern Brazil. Porto Alegre. Esc. Geol. Publ. Esp., 15:1-51.
CLOSS, D. \& MEDEIROS, V. M. F. 1965. New observations on the ecological subdivision of the Patos lagoon in southern Brazil. Bol. I.C.N., 24. 35 p 1pl.

\& 1967. Thecamoebina and foraminifera from the Mirim lagoon Southern Brazil. Porto Alegre. Iheringia, Zool., 35:75-88.

DEBENAY, J. P. 1990. Recent foraminiferal assemblages and their distribution relative to environmental stress in the paralic environments of west Africa (Cape Timiris to Ebric lagoon). J. foram. Res., 20(3):267-282.

; COLLEUIL, B. \& TEXIER H. 1993. Peuplements de foraminiferes du lac Nokoué (Bénin) avant fermeture de la lagune. Comparaison avec d'autres environnements paraliques d'Afrique de l'Ouest. Revue Micropalćont., 36(2):225-234.

FORTI, R. S. \& ROETTGER, E. 1967. Further observations on the seasonal variations of mixohalline foraminifera from the Patos lagoon. Southern Brazil. Venise. Arch. Oceanogr. Limmol., 15:55-61.

KUTNER, M. B. B. \& AIDAR-ARAGĀO, E. 1986. Influência do fechamento do Valo Grande sobre a composição do fitoplâncton na regiāo de Cananéia $\left(25^{\circ} \mathrm{S}-48^{\circ} \mathrm{W}\right)$. In: SIMPÓSIO INTERNACIONAL ALGAS: A ENERGIA DO AMANHĀ, São Paulo, 1982. Anais. Bicudo, C. E.; Tcixera, C. \& Tundisi, G., eds Sāo Paulo, Instituto Oceanográfico, Universidade de Sāo Paulo, Brasil. p.109-120.

MADEIRA, M. L. 1969. Foraminifera from Sao Francisco do Sul State of Santa Catarina. Brasil. Iheringia. Zool., Porto Alegre, 37:3-29.

MADEIRA-FALCETA, M. 1974. Ecological distribution of the Thecamoebal and Foraminiferal Associations in the Mixohaline Environments of the Southern Brazilian littoral. An. Acad. bras. Ciênc., 46(3/4):667-687.

MARTIN, L.; SUGUIO, K. \& FLEXOR, J.-M. 1984. Informaçōes fornecidas pelos sambaquis na reconstrução de paleolinha de praia quaternária: exemplos da costa brasileira. Revta Pré-Hist., 6:128-147. 
MARTIN, L.; SUGUIO, K. \& FLEXOR, J.-M. 1987. Flutuaçōes do nível relativo do mar no Ouaternário e seu papel na sedimentação costeira: exemplos brasileiros. In: SIMPÓSIO SOBRE ECOSSISTEMAS DA COSTA SUL E SUDESTE BRASILEIRA, SÍNTESE DOS CONHECIMENTOS. Cananéia, 1987. São Paulo, ACIESP, 54(1):40-61.

MISHIMA, M.; YAMANAKA, N.; PEREIRA, O. M.; SOARES, F. das C. SINOUE, C.; AKABOSHI, S. \& JACOBSEN, O. 1985. Hidrografia do Complexo Estuarino-Lagunar de Cananéia $\left(25^{\circ} \mathrm{S}-48^{\circ} \mathrm{W}\right)$ São Paulo, Brasil. I - Salinidade e temperatura (1973 a 1980). Bolm Inst. Pesca, São Paulo, 12:109-121.

MURRAY, J. W. 1973. Distribution and ecology of living benthic Foraminiferids. Heinemann Educational Books, Londres, 274 p.

MIYAO, S. Y.; NISHIHARA, L. \& SARTI C. C. 1986. Características físicas e químicas do sistema estuarino-lagunar de Cananéia- Iguape. Bolm Inst. oceanogr., S Paulo, 34(1):23-36.

PETRI, S. 1971. O gênero Ammonia e as correlaçōes dos sedimentos da parte superior do Cenoź́ico da faixa litorânea brasilcira. An. Acad. bras. Ciênc., 43(supl.):557-575.

\& SUGUIO, K. 1973. Stratigraphy of the Iguape-Cananéia lagoonal region sedimentary deposits. Sao Paulo State, Brasil. Part II. Heavy mineral studies, microorganisms inventories and stratigraphical interpretations. Bolm IG, Inst. Geociências, Univ. S Paulo, 4:71-85.

POR, F. D.; ALMEIDA PRADO POR, M. S. \& OLIVEIRA, E. C. 1984. The mangal of the estuary and lagoon sustem of Cananéia (Brazil). In: Por, F. D. \& Dor I. eds Hydrobiology of the Mangal. The ecosystem of the mangrove forests. The Hague, W. Junk Publishers, p.211-228.

SAUNDERS, J. B. 1958. Recent foraminiferida of mangrove swamps and river estuaries and their fossil counterparts in Trinidad. Micropaleont. 4(1):79-92.

SCHAFER, C. T. \& SEN GUPTA, B. K. 1969. Foraminiferal ccology in polluted estuaries of New Brunswick and Maine. Atlant. Oceanogr. Lab. Rpt., Dartmouth, 6(3):121-134.
SCHAEFFER-NOVELLI, Y.; MESOUITA, H. de S. L. \& CINTRON-MOLERO, G. 1990. The Cananéia lagoon estuarine system, São Paulo, Brazil. Estuaries, Columbia, 13(2):193-203.

SCOTT, D. B. \& MEDIOLI, F. S. 1980. Living Vs. Total foraminiferal populations: Their relative usefulness in paleoecology. J. Paleont., 54(4):814-831.

; SCHNACK, E. J.; FERRERO, L.; ESPINOSA, M. \& BARBOSA, C. F. 1990. Recent marsh foraminifera from the east coast of South America. Comparison to the northern Hemisphere. In: Hemlehen, C. et al. eds Paleoecology, Biostratigraphy, Paleoceanography and taxonomy of aglutinated foraminifera. In: WORKSHOP ON AGLUTINATED FORAMINIFERA, 3., Tübingen, 1989. p.717- 737.

SHARIFI, A. R.; CROUDACE, I. W. \& AUSTIN, R. L. 1991. Benthic foraminiferids as pollution indicators in Southampton Water, southern England, U.K. J. Micropaleontology, Londres, 10:(1):109-113.

SUGUIO, K.; MARTIN, L. \& FLEXOR, J.-M. 1976. Les variations relatives du niveau moyen de la mer au Quaternaire récent dans la région de Cananéia-Iguape (Sâo Paulo). Bolm IG, Inst. Geociênc., Univ. S Paulo, 7:113-129.

\& TESSLER, M. G. 1983. Intense coastal sedimentation and erosion in the Cananeia outlet, southern state of São Paulo, Brazil. Bolm Inst. oceanogr., S Paulo, 32(1):71-75.

TESSLER, M. G. \& MAHIQUES, M. M. de 1993. Utilization of coastal geomorphic features as indicators of longshore transport: Examples of the southern coastal region of the state of São Paulo, Brasil. J. coast. Res., 9(3):823-830.

TINOCO, I. de M. 1958. Observaçāo sobre a microfauna de foraminíferos da Lagoa de Araruama, Estado do Rio de Janeiro. An. Acad bras Ciênc., 30(4):575-584.

1966. Contribuiçāo a sedimentologia e microfauna da Baía de Sepetiba (Estado do Rio de Janeiro). 2: Foramininíferos. Trabhs. Inst. Oceanogr.. Univ. Fed. Pernambuco, Recife, (7/8):123-133. 
TUNDISI, J. G. 1981. Ecological studies of the lagunar region of Cananéia: a review. In: Martin, J. M., Burton, J.D. \& Eisma D., eds River inputs to Ocean Systems. UNEP/IOC/SCOR. UNEP/UNESCO, p.298-304.

WALTON, W. R. 1952. Techniques for recognition of living foraminifera. Contr. Cushman.Fnd. form. Res., 3(2):56-60.

ZANINETTI, L.; BRÖNNIMANN, P.; BEURLEN, G. \& MOURA, J. A. 1977. La mangrove de Guaratiba et la Baía de Sepetiba, État de Rio de Janeiro, Brésil. Foraminifères et Ecologie. Archs Sci., Genève, 30(2):161-178.
ZANINETTI, L.; BRÖNNIMANN, P.; DIAS-BRITO, D.; ARAI, M., CASALETTI, P.; KOUTSOUKOS, E. \& SILVEIRA, S. 1979. Distribution écologique des foraminifères dans la mangrove d'Acupe, État de Bahia, Brésil. Notes Lab. palaeont., Univ. Genève, 4(1):1-17.

(Manuscrit réception 10 Février 1994; corrigé 05 Octobre 1994; agrée 22 Mai 1995) 